\title{
Comparación de dos comunidades biológicas marinos costeras en Cedeño, Choluteca
}

\author{
Marco Herminio Osorto, Emalis Ulloa Martinez, Fernanda Gabriela Alvarez, \\ Evelin Alejandra Rodriguez ,Fredy Adolfo Gimenez ${ }^{1}$
}

Vanessa Merlo ${ }^{2}$

\section{RESUMEN}

La investigación tiene como objetivo comparar dos zonas de muestreo en la abundancia de especies marinos costeras en Estero Cabrera Punto A y Estero Tamasil punto $B$, así mismo, los objetivos específicos se orientaron en determinar la abundancia y densidad de especies marino costeras, de igual manera establecer la relación de parámetros biofísicos con los grupos taxonómicos colectados. La ubicación de las zona comparada se realizó en la comunidad costera de Cedeño a una latitud $13^{\circ} 10^{\prime} 11.54 " \mathrm{~N}$ y una longitud $87^{\circ} 25^{\prime} 41.48^{\prime \prime} \mathrm{W}$, en el municipio de Marcovia a $39.3 \mathrm{~km}$ de la ciudad de Choluteca, los días 9 y 10 de septiembre del 2016.

Durante la colecta de organismos, se efectuaron dos cuadrantes de $5 \times 5$ metros en el punto A Estero Cabrera el día viernes 9 de septiembre del 2016 y en el día sábado 10 de septiembre del 2016 en el punto B Estero Tamasil. Consecuentemente para cada cuadrante se tomaron coordenadas y parámetros biofísicos.

Los resultados encontrados en el Punto $A$, fueron 7 individuos y 3 familias taxonómicas, en el Punto B, 5 individuos y 4 familias taxonómicas. Dentro de la clasificación punto A, se encontraron los siguientes filos: Molusca Y Artrópoda. Punto B: Artrópoda y Chordata. Con un total de 12 individuos colectados. Tal comparación de las comunidades se utilizó: Índice de Shannon, Índice de Simpson, Densidad absoluta y Densidad Relativa, Moda, Media y Mediana.

Palabras Claves: Comparación, Estero Cabrera, Estero Tamasil, Abundancia, Densidad, Índice de Shannon, Índice de Simpson, Moda, Media, Mediana.

\footnotetext{
${ }^{1}$ Estudiantes de la Carrera de Ingeniería acuícola y recursos marinos, Centro Regional Universitario del Litoral Pacífico (CURLP-UNAH)

${ }^{2}$ Profesora de Genética Médica y Biología General, Departamento de Biología General y Genética, Escuela de Biología, Facultad de Ciencias-UNAH: vanessa.rodriguez@unah.edu.hn
} 


\section{ABSTRACT}

The research aims to compare two sample areas of the coastal marine species in Estero Cabrera Point A and Estero Tamasil point B. One of the specific objectives was to determine the abundance and density of coastal marine species, and therefore, establish the relationship between biophysical parameters and the collected taxonomic groups. The location of the compared zone was made in the coastal community of Cedeño at a latitude $13^{\circ} 10^{\prime} 11.54$ " $\mathrm{N}$ and a length $87^{\circ} 25^{\prime} 41.48$ " W, in the municipality of Marcovia, $39.3 \mathrm{~km}$ from the city of Choluteca, September 9 and 10, 2016.

During the collection of organisms, the sample areas consisted in two quadrants of 5 x 5 meters located at point A Estero Cabrera on Friday, September 9, 2016 and on Saturday, September 10, 2016 at point B Estero Tamasil. Consequently, biophysical coordinates and parameters were taken for each quadrant.

The results found in Point A were 7 individuals and 3 taxonomic families, and in Point $B, 5$ individuals and 4 taxonomic families. Within the classification points, the following phyla were found: in Point A (Mollusk and Arthropoda) and in Point B (Arthropoda and Chordata), with a total of 12 individuals collected. Such comparison of communities was used: Shannon Index, Simpson Index, absolute density and relative density, fashion, average and median.

Keywords: Coastal marine species, Estero Cabrera, Estero Tamasil, Shannon Index, Simpson Index. 


\section{INTRODUCCION}

Un ecosistema es un sistema ecológico con componentes biológicos que interactúan formando así una comunidad biológica. Esta comunidad biológica con diferentes poblaciones compuestas de diferentes organismos está relacionada de manera inseparable con su ambiente físico. (Hart, 2014, pág. 9)

Los ecosistemas acuáticos costeros son cuerpos de agua que se encuentran en la zona litoral manteniendo una comunicación permanente o temporal con el mar y que puede o no estar conectados con sistemas dulceacuícolas. Esto hace que el tipo de salinidad vaya desde salobre hasta típicamente marina. En este ecosistema se encuentran los esteros que se han formado de un canal natural o de antiguos brazos de un delta de rio actualmente cerrado, en ellos se presentan riqueza y diversidad de especies formando comunidades biológicas (O. sanchez, 2007, pág. 39)

La riqueza y diversidad de especies son propiedades emergentes de las comunidades biológicas y comúnmente son utilizadas para describir una taxocenosis, para determinar su distribución espacial y presencia. Debido a que la diversidad biológica sirve como base para explicar la estructura y función de las comunidades. (Bojorges-Baños, 2011, p. 205-206)

Es importante reconocer que la diversidad tiene varios componentes correlacionados, entre los cuales se encuentran el más ampliamente reconocido, medido por el número de especies presentes en un hábitat, al cual llamamos riqueza o diversidad de especies, y un segundo componente llamado diversidad funcional, el cual es medido por el rango de atributos de las especies en un hábitat dado. (Saldaña, 2013, p. 178).

Las comunidades biológicas responden constantemente a los cambios físicos, a la dinámica química y procesos eco sistémico por lo que las diferencias locales y regionales en la hidrología, geología y disponibilidad de nutrientes en el sistema, promueven escenarios espaciales complejos en las interacciones de las comunidades generando efectos directos e indirectos sobre la trama trófica.(Graciela Ferrari, 2012, p. 5). "La zona litoral es el ecosistema más cercano a la costa. Es una zona de transmisión entre la tierra y el océano, llamada zona fótica por la presencia del ion fosfato, es una zona superficial de dominio pelágica que se encuentra bien iluminada y ahí encontramos ecosistemas como ser de algas, moluscos, equinodermos, y otros." (Camposbedolla, 2003, pág. 143) 
"En el ecosistema perteneciente al Filo Molusca es de los filos más grandes después de los artrópodos, por lo que incluyen varias especies. Su cuerpo es blando, pero también son bastantes conocidos por la gran variedad de sus conchas." (Lizarralde, 2015, pág. 17).

La clase bivalvos como su nombre lo indica, su concha se caracteriza por estar compuesta de 2 valvas laterales de forma, tamaño y color variable. Su cuerpo se encuentra encerrado en un manto bilobulado, cabeza reducida y pie en forma de cuña. (Lizarralde, 2015, pág. 21)

Los moluscos constituyen uno de los principales grupos de organismos que conforman ensambles estructuradores de comunidades intermareales. Junto a las macro algas, aportan la mayor riqueza específica de estos ambientes y muchos de ellos proveen de sustrato a otras especies. Constituyen uno de los principales grupos de consumidores tanto carnívoros como herbívoros, modelando la estructura comunitaria del intermareal. A su vez, son sensibles a cambios ambientales de carácter estocástico o periódico, como cambios estacionales por fotoperiodo.(Jaime Ojeda, 2014, p. 494).

La costa centroamericana se caracteriza por una alta diversidad fisiográfica dadas sus numerosas penínsulas, golfos y bahías. Los recursos marinos vivos constituyen una importante fuente de proteínas y su utilización es de importancia comercial y económica para la región. En la zona costera de la región se crían varias especies con fines comerciales tales como tilapia, bivalvos, crustáceos, entre otros. La más importante desde el punto de vista económico, social y ambiental es la cría de camarón blanco (Litopenaeus Vannamei). (José Joaquín Rodríguez, 1998, págs. 9, 13,14) En el Golfo de Fonseca se integran varios ecosistemas y ambientes costeros que hacen propicio el mantenimiento de una alta diversidad de organismos, el grupo de crustáceos son los más abundantes en cuanto a especies marinas; la cual la mayoría de especies presenta un alto consumo de estos, específicamente de camarones peneidos, seguido por peces y en menor grado de moluscos. (Francisco Batres, 2012, págs. 24, 26)

Los crustáceos se distribuyen en todos los esteros, sobre todo abundan poslarva de las especies de camarón blanco (Litopenaeus Vannamei). (Secretaría de Recursos Naturales y Ambiente de Honduras.Ministerio de Medio Ambiente y Recursos Naturales de El Salvador, 1998, pág. 36) 


\section{MATERIALES Y METODOS}

\section{Área de Estudio}

La zona seleccionada para la investigación, fue en Playa Cedeño, municipio de Marcovia departamento de Choluteca ubicado a $39.3 \mathrm{~km}$ del sur de Marcovia. Áreas de influencia: Punta Ratón, El Venado y Boca de Rio Viejo, considerados sitio precedente de comunidades biológicas. Dentro de las siguientes actividades (playa Cedeño) se realizaron 2 cuadrantes en los siguientes puntos: Punto A, Estero Cabrera $13^{\circ} 09.39 .2 \mathrm{~N} 087^{\circ} 25.71 .0^{\prime} \mathrm{O}$ (9 de septiembre de 2016). Punto B, Estero Tamasil $13^{\circ} 09.452 \mathrm{~N} 087^{\circ} 25.753^{\prime} \mathrm{O}$ (10 de septiembre de 2016).

\section{DISEÑO METODOLÓGICO}

El alcance de la investigación es descriptivo con un enfoque cuantitativo. El estudio, consistió en la comparación y ubicación de dos comunidades biológicas marino costeras en Estero Cabrera (punto A) y Estero Tamasil (punto B) realizados en fechas 9 y 10 de septiembre. Para la recolección de datos se establecieron 3 operaciones: Toma de parámetros biofísicos (Temperatura y pH) del agua, para saber condiciones ambientales en las que se encuentra las especies recolectadas en cada punto; Toma de las coordenadas utilizando el instrumento apropiado (receptor de GPS), Además se efectuó el conteo de individuos y se determinó la biometría de los organismos colectados.

Cada área de estudio está ubicada entre cada una a la distancia de 50 metros. Cada cuadrante se midió 5 metros $\times 5$ metros. Para proceder a realizar la colecta de organismos se efectuó la comparación de las mareas mediante la observación las horas de colecta se realizaron en marea baja; punto A (Hora: 1:59pm) y Punto B (Hora: 2:49). Para el análisis de los datos se emplearon el índice de diversidad, índice de Simpson, densidad y las medidas de tendencia central, como moda, media y mediana.

"Índice de diversidad de especies donde: $\mathrm{N}^{\circ}$ de individuos/Total de individuos del total de especies." 


\section{Figura 1. Formula Indicé de Shannon}

$$
H^{\prime}=-\sum_{i=1}^{S} P_{i} \ln \left(P_{i}\right)
$$

Dónde:

$S=$ número de especies

$\mathrm{Pi}=$ proporción de individuos de la especie $\mathrm{i}$

A mayor valor de H' mayor diversidad de especies.

Índice de Simpson: $\mathrm{N}^{\circ}$ de individuos por especie/total de individuos de todas las especies, donde el total de abundancia relativa x especie elevada al cuadrado.

Fuente: (VM Zarco-Espinosa, 2010, pág. 6)

\section{Figura 2. Formula Indicé de Simpson}

$$
S=\frac{1}{\sum \frac{n_{i}\left(n_{i}-1\right)}{N(N-1)}}
$$

Dónde:

ni = número de individuos en la iésima especie

$\mathrm{N}=$ número total de individuos

A mayor valor de $\mathrm{S}$ menor dominancia de una (o de un grupo) de especie(s).

También, se calcularon los individuos dentro del cuadrante que se repiten, mayor frecuencia y mediana.

Densidad relativa:

$$
\frac{\text { Densidad absoluta por cada especie } \mathrm{X} 100}{\text { Densidad absoluta de todas las especies }}
$$

Densidad absoluta:

Numero de individuo de especies.

$$
\text { Área muestreada }
$$


Moda: Mo

Media: $\bar{X}=\frac{X 1+X 2+X 3 \ldots X 4}{N}$

Mediana: M

Fuente: (VM Zarco-Espinosa, 2010)

Figura 3. Área de Estudio, Estero Cabrera y Estero Tamasil

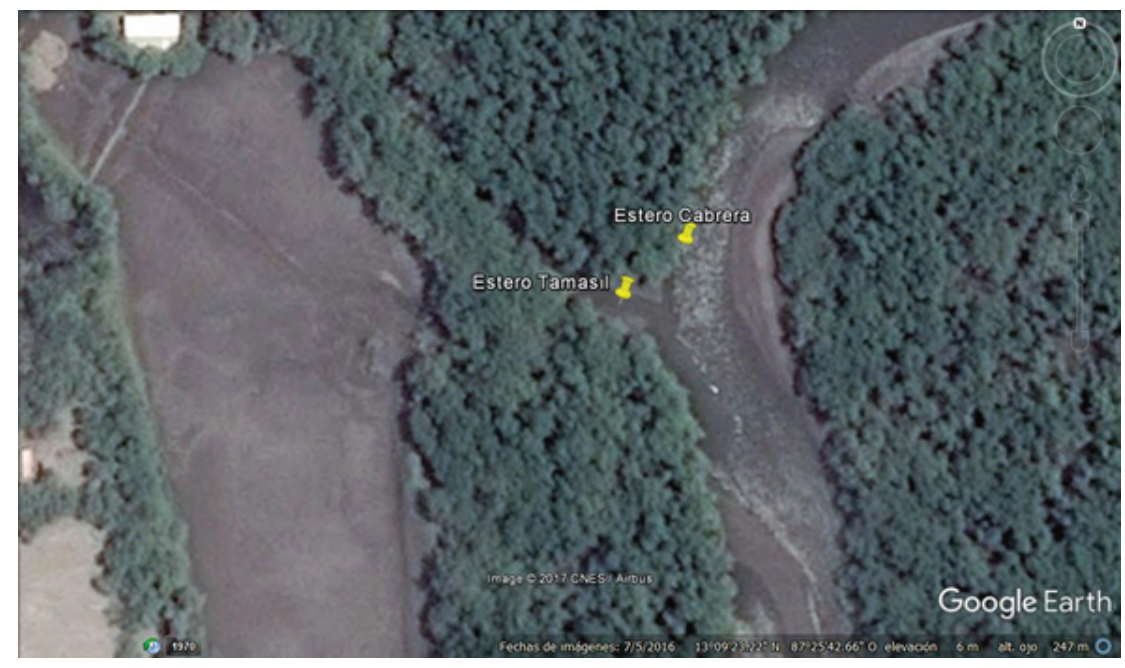

Fuente: (earth, 2017)

Figura 4. Especificaciones de los cuadrantes. Punto A (Estero Cabrera), Punto B, (Estero Tamasil)

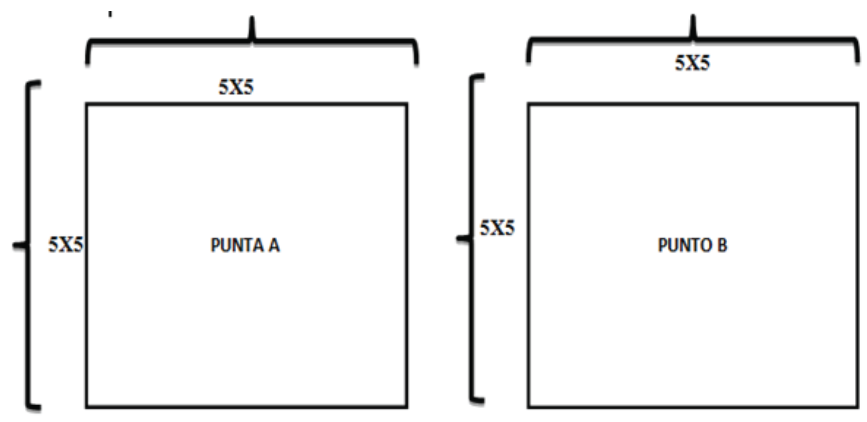

Fuente: Elaboracion Propia) 


\section{RESULTADOS}

Los días 9 y 10 de septiembre de 2016 se inició mediante toma de datos biofísicos, toma de coordenadas, colecta de organismos marinos 3:00 pm, finalizando a las 5:30 pm.

Tabla 1. Organismos recolectados en Estero Cabrera (Punto A 9 de septiembre)

\begin{tabular}{|c|c|c|c|}
\hline $\mathbf{N}^{\circ}$ & $\mathbf{N}^{\circ}$ de Individuos & $\begin{array}{c}\text { Clasificación Taxonómica } \\
\text { Familo: Molusca } \\
\text { FamCIDAE }\end{array}$ & Anadara tuberculosa \\
\hline 2 & 3 & $\begin{array}{c}\text { Filo: Molusca } \\
\text { Familia: DONACIDAE }\end{array}$ & Iphigenia agior \\
\hline 3 & 2 & $\begin{array}{c}\text { Filo: Artrópoda } \\
\text { Familia: OCYPODIDAE }\end{array}$ & Uca sp. \\
\hline
\end{tabular}

Fuente: Elaboración propia

Tabla 2. Organismos Recolectados en Estero Tamasil (Punto B 10 de septiembre)

\begin{tabular}{|c|c|c|c|}
\hline $\mathrm{N}^{\circ}$ & $\mathrm{N}^{\circ}$ de Individuos & Clasificación Taxonómica & Nombre Cientifico \\
\hline 1 & 1 & $\begin{array}{c}\text { Filo: Artrópoda } \\
\text { Familia: PENAIDEA }\end{array}$ & Litopenaeus Vannamei \\
\hline 2 & 1 & $\begin{array}{c}\text { Filo: Artrópoda } \\
\text { Familia : XANTIDAE }\end{array}$ & Ocypode sp. \\
\hline 3 & 1 & $\begin{array}{c}\text { Filo: Chordata } \\
\text { Familia: CLUPEIDAE }\end{array}$ & Sardinops sp. \\
\hline 4 & 1 & $\begin{array}{c}\text { Filo: Chordata } \\
\text { Familia: ATHERINIDAE }\end{array}$ & Antherinella sp. \\
\hline 5 & 1 & $\begin{array}{c}\text { Filo: Chordata } \\
\text { Familia: CLUPEIDAE }\end{array}$ & Etrumeos sp. \\
\hline
\end{tabular}

Fuente: Elaboración propia 
Tabla 3. Organismos recolectado en Punto A y Punto B

\begin{tabular}{|c|c|c|c|}
\hline Puntos & Moluscos & Artrópodos & Chordata \\
\hline Estero Cabrera (Punto A) & $5(100 \%)$ & $2(50 \%)$ & $0(0 \%)$ \\
\hline Estero Tamasil (Punto B) & $0(0 \%)$ & $2(50 \%)$ & $3(100 \%)$ \\
\hline
\end{tabular}

Fuente: Elaboración propia

Tabla 4. Índice de Simpson. Estero Cabrera (punto A)

\begin{tabular}{|c|l|c|c|c|}
\hline $\mathbf{N}^{\circ}$ & Especie & Abundancia & $\mathbf{p}$ & $\mathbf{p 2}$ \\
\hline 1 & Anadara tuberculosa & 1 & 0.428571428 & 0.183673469 \\
\hline 2 & Iphigenia agior & 2 & 0.285714285 & 0.081632653 \\
\hline 3 & Uca & 3 & 0.285714285 & 0.081632653 \\
\hline Total & & 7 & 0.999999998 & 0.346938775 \\
\hline S & & & & 0.653061225 \\
\hline
\end{tabular}

Fuente: Elaboración propia

Tabla 5. Índice de Simpson. Estero Tamasil (Punto B).

\begin{tabular}{|c|c|c|c|c|}
\hline $\mathbf{N}^{\circ}$ & Especie & Abundancia & $\mathbf{p}$ & $\mathbf{p 2}$ \\
\hline 1 & Litopenaeus Vannamei & 1 & 0.2 & 0.04 \\
\hline 2 & Ocypode sp. & 1 & 0.2 & 0.04 \\
\hline 3 & Sardinops sp. & 1 & 0.2 & 0.04 \\
\hline 4 & Antherinella & 1 & 0.2 & 0.04 \\
\hline 5 & Etrumeos & 1 & 0.2 & 0.04 \\
\hline Total & & 5 & 1 & 0.2 \\
\hline S & & & 0.8 & 0.8 \\
\hline
\end{tabular}

Fuente: Elaboración propia 
Tabla 6. Calculo de Índice de Shannon. Estero Cabrera (Punto A)

\begin{tabular}{|l|l|l|l|l|c|}
\hline $\mathbf{N}^{\circ}$ & Especie & $\mathrm{I}$ & $\mathrm{Pi}$ & $\ln (\mathrm{pi})$ & $\mathrm{Pi}$ in(pi) \\
\hline 1 & Anadara tuberculosa & 3 & 0.428571428 & -0.847297861 & -0.363127654 \\
\hline 2 & Iphigenia agior & 2 & 0.285714285 & -1.252762971 & -0.357932276 \\
\hline 3 & Uca & 2 & 0.285714285 & -1.252762971 & -0.357932276 \\
\hline Total & & 7 & 0.99999998 & -3.352823803 & -1.078992207 \\
\hline $\mathrm{H}^{\prime}$ & & & & & 1.078992207 \\
\hline
\end{tabular}

Fuente: Elaboración propia

Tabla 7. Calculo de Índice de Shannon. Estero Tamasil (Punto B)

\begin{tabular}{|l|l|l|l|l|c|}
\hline$N^{\circ}$ & Especie & I & Pi & In(pi) & Pi $\ln (\mathrm{pi})$ \\
\hline 1 & Litopenaeus Vannamei & 1 & 0.2 & -1.609437912 & -0.321887582 \\
\hline 2 & Ocypode sp. & 1 & 0.2 & -1.609437912 & -0.321887582 \\
\hline 3 & Sardinops sp. & 1 & 0.2 & -1.609437912 & -0.321887582 \\
\hline 4 & Antherinella & 1 & 0.2 & -1.609437912 & -0.321887582 \\
\hline 5 & Etrumeos & 1 & 0.2 & -1.609437912 & -0.321887582 \\
\hline Total & & 5 & 1 & -8.047189562 & -1.609437912 \\
\hline$H^{\prime}$ & & & & & 1.609437912 \\
\hline
\end{tabular}

Fuente: Elaboración propia

Tabla 8. Densidad Relativa. Estero Cabrera (Punto A)

\begin{tabular}{|c|c|c|c|c|}
\hline $\mathbf{N}^{\circ}$ & $\begin{array}{l}\mathbf{N}^{\circ} \text { de organismos } \\
\text { por especie }\end{array}$ & Organismos & D. Absoluta & D. Relativa \\
\hline 1 & 3 & Anadara tuberculosa & 0.6 & 42.85714286 \\
\hline 2 & 2 & Iphigenia agior & 0.4 & 28.57142857 \\
\hline 3 & 2 & Uca & 0.4 & 28.57142857 \\
\hline Total & & & 1.4 & 100 \\
\hline
\end{tabular}

Fuente: Elaboración propia 
Tabla 9. Densidad Relativa. Estero Tamasil (Punto B)

\begin{tabular}{|c|c|c|c|c|}
\hline$N^{\circ}$ & $\begin{array}{l}\mathbf{N}^{\circ} \text { de organismos } \\
\text { por especie }\end{array}$ & Organismos & D. Absoluta & D. Relativa \\
\hline 1 & 1 & $\begin{array}{c}\text { Litopenaeus } \\
\text { Vannamei }\end{array}$ & 0.2 & 20 \\
\hline 2 & 1 & Ocypode $s p$ & 0.2 & 20 \\
\hline 3 & 1 & Sardinops sp. & 0.2 & 20 \\
\hline 4 & 1 & Antherinella & 0.2 & 20 \\
\hline 5 & 1 & Etrumeos & 0.2 & 20 \\
\hline Total & 5 & & 1 & 100 \\
\hline
\end{tabular}

Fuente: Elaboración propia

Tabla 10. Moda, Media y Mediana. Punto A y Punto

\begin{tabular}{|c|c|c|c|}
\hline Puntos & Moda & Media & Mediana \\
\hline Estero Cabrera (Punto A) & 2 & 3.5 & 4 \\
\hline Estero Tamasil (Punto B) & 1 & 2.5 & 3 \\
\hline
\end{tabular}

Fuente: Elaboración propia

\section{Tabla 11. Parámetros Biofísicos}

\begin{tabular}{|c|c|c|}
\hline Parámetros & Estero Cabrera & Estero Tamasil \\
\hline $\mathrm{pH}$ & 7.04 & 7.06 \\
\hline Temperatura & $35.3^{\circ} \mathrm{C}$ & $34^{\circ} \mathrm{C}$ \\
\hline
\end{tabular}

Fuente: Elaboración propia 
Figura 5. Organismos Encontrados en Punto A y Punto B

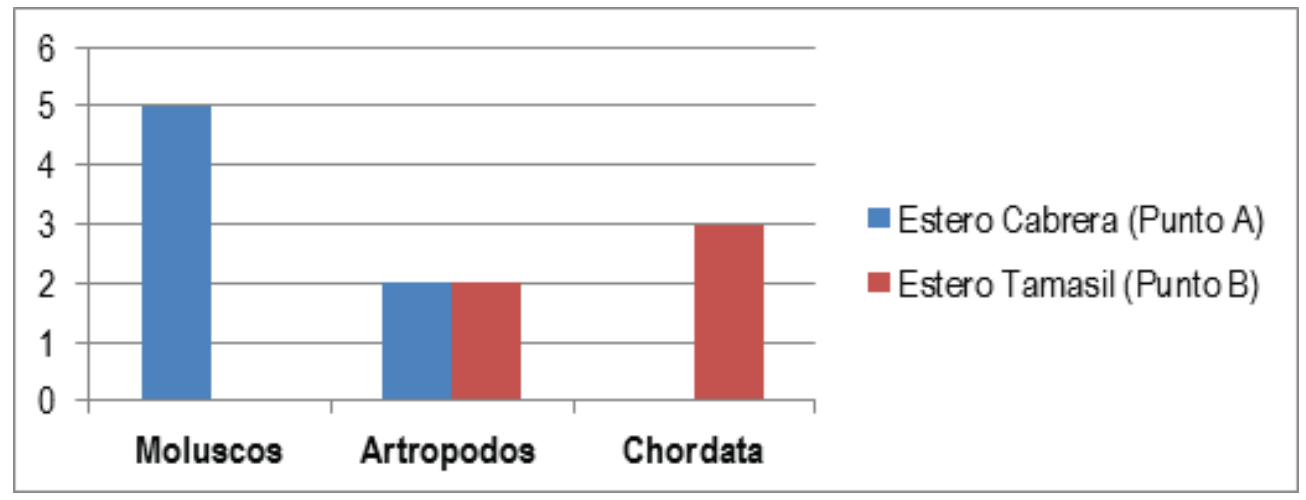

Fuente: Elaboración propia

Figura 6. Índice de Simpson. Punto A

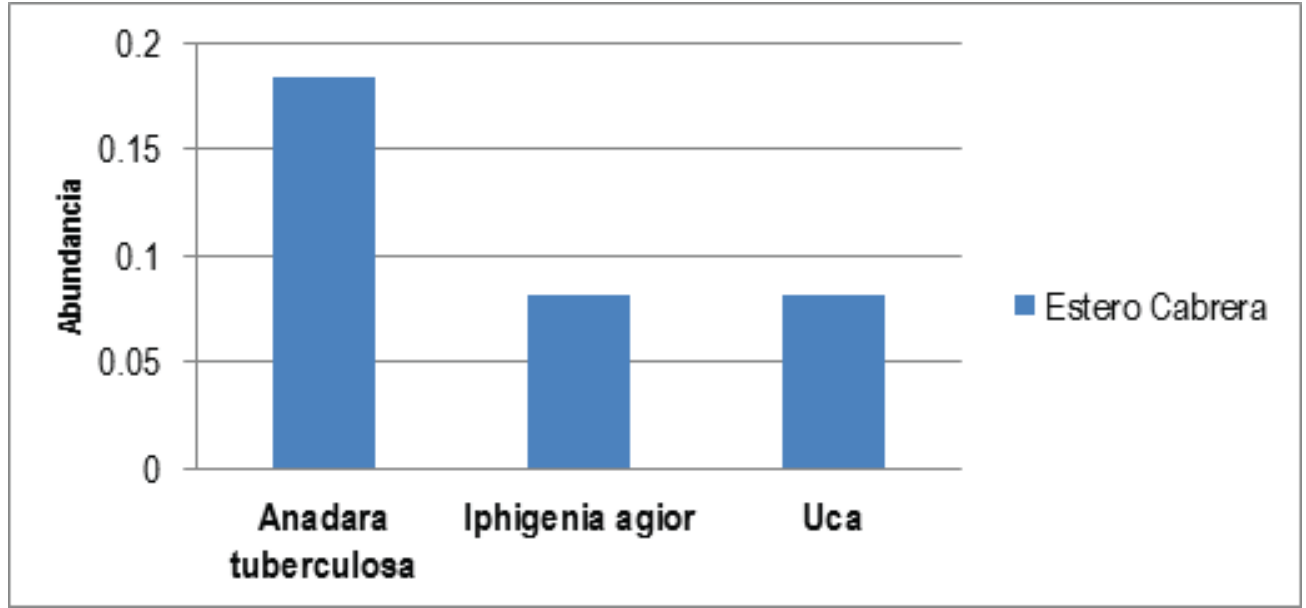

Fuente: Elaboración propia

Figura 7. Índice de Simpson. Punto B

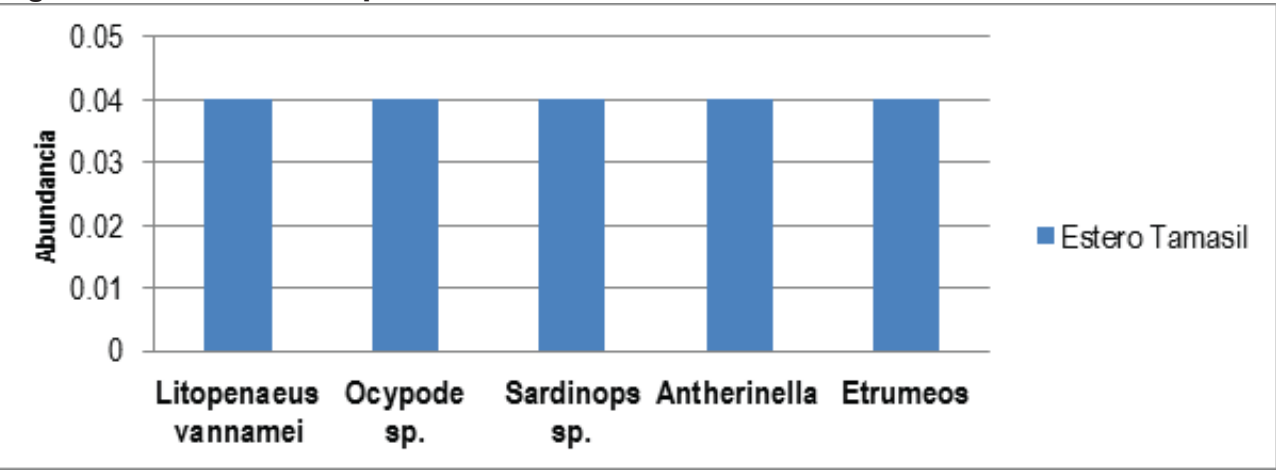

Fuente: Elaboración propia 
Figura 8. Índice de Shannon. Punto A

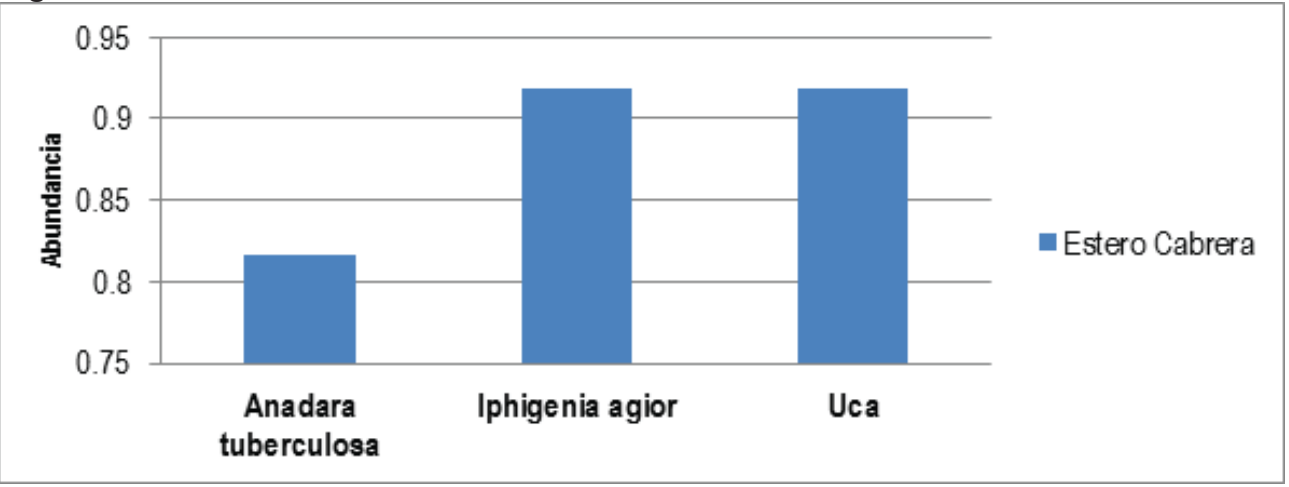

Fuente: Elaboración propia

Figura 9. Índice de Shannon. Punto B

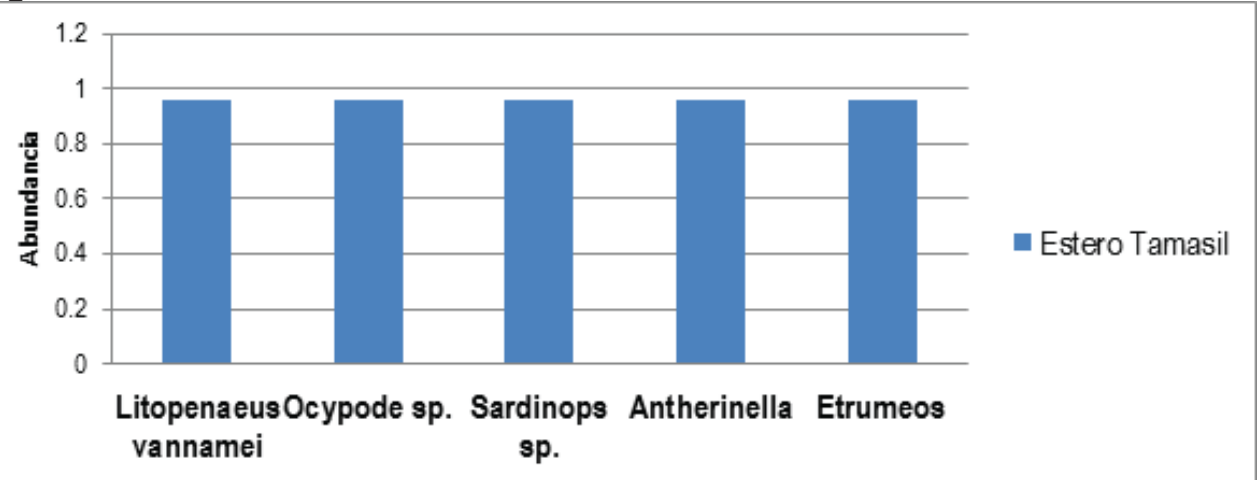

Fuente: Elaboración propia

Figura 10. Densidad Absoluta y Relativa. Punto A

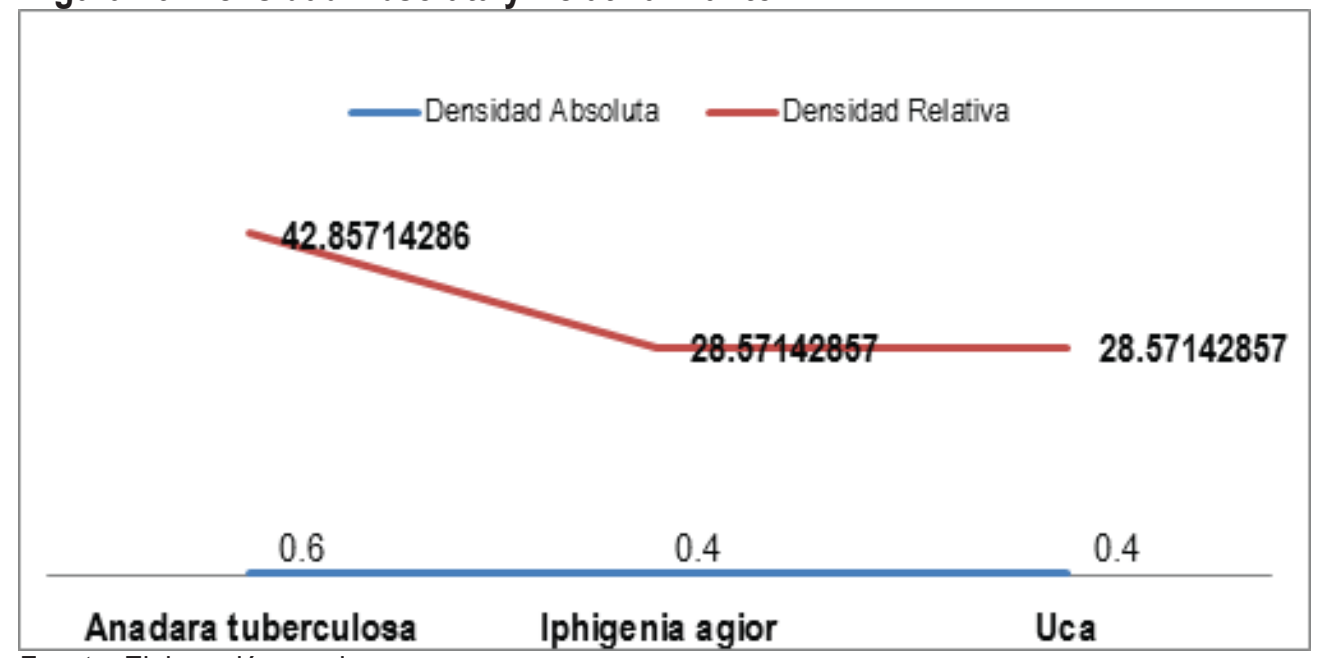

Fuente: Elaboración propia 
Figura 11. Densidad Absoluta y Relativa. Punto B

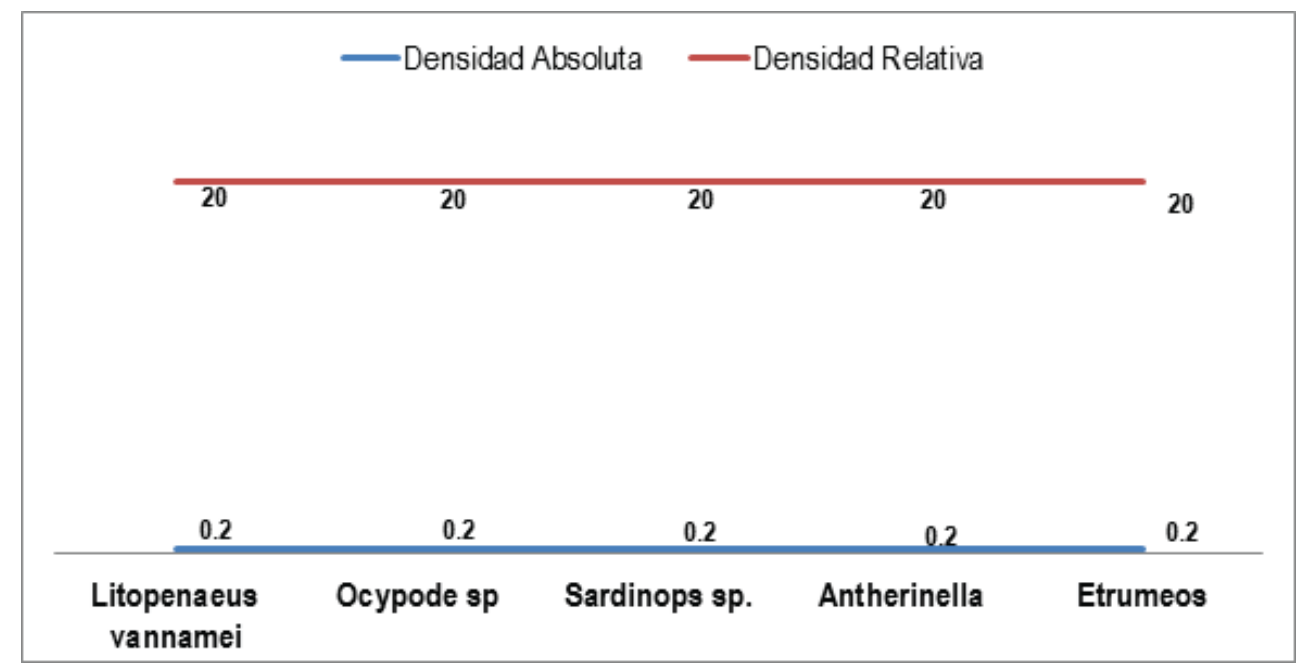

Fuente: Elaboración propia

Figura 12. Parámetros Biofísicos, Estero Cabrera y Estero Tamasil.

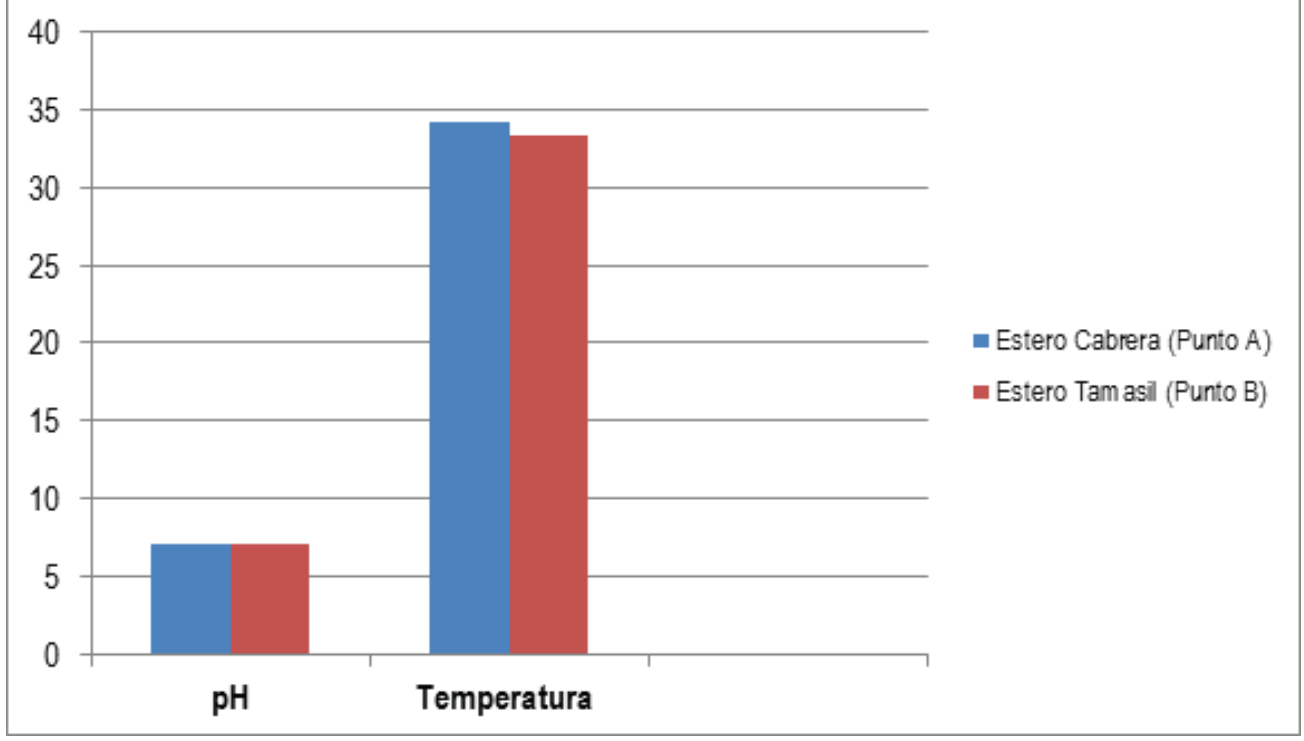

Fuente: Elaboración propia 
Figura 13. Organismos Recolectados en Estero Cabrera (Punto A) Fuente: Fotos tomadas por Grupo de la clase de Biología Marino Costero II periodo del año 2016.

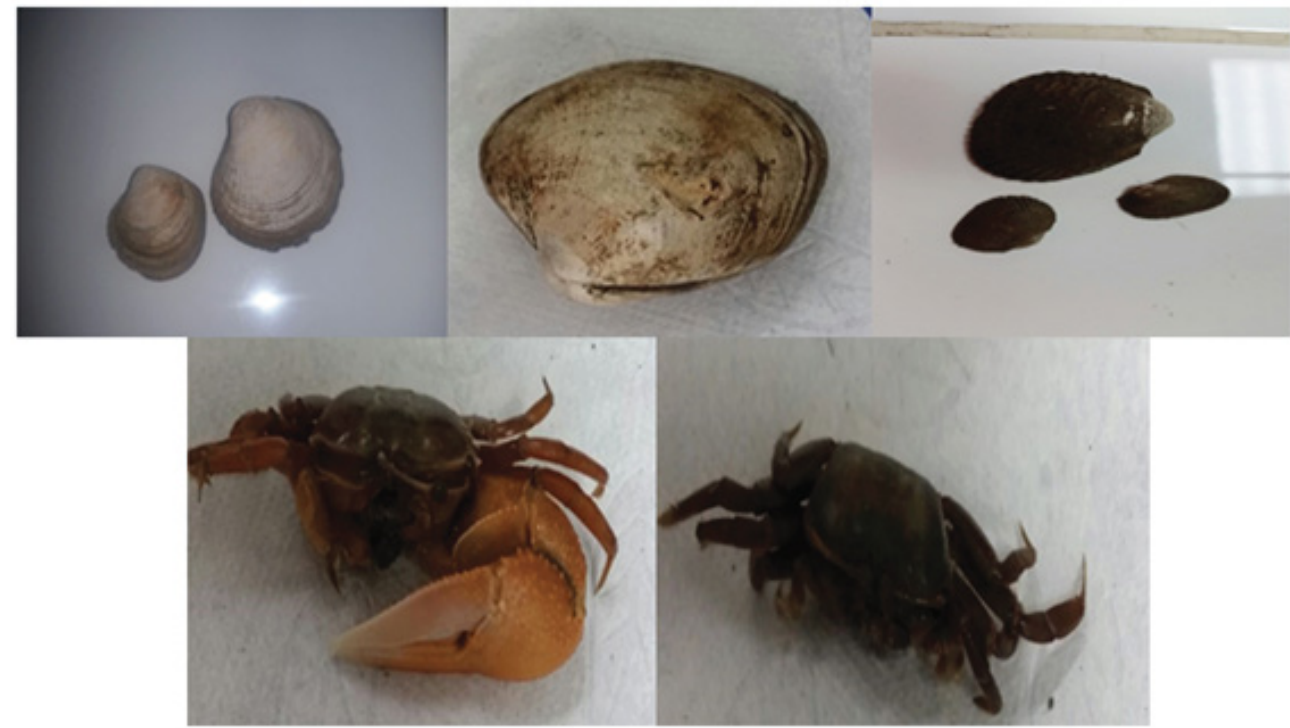

Fuente: Elaboración propia

Figura 14. Organismos Recolectados en Estero Tamasil (Punto B) Fuente: Fotos tomadas por Marco Nuñez.

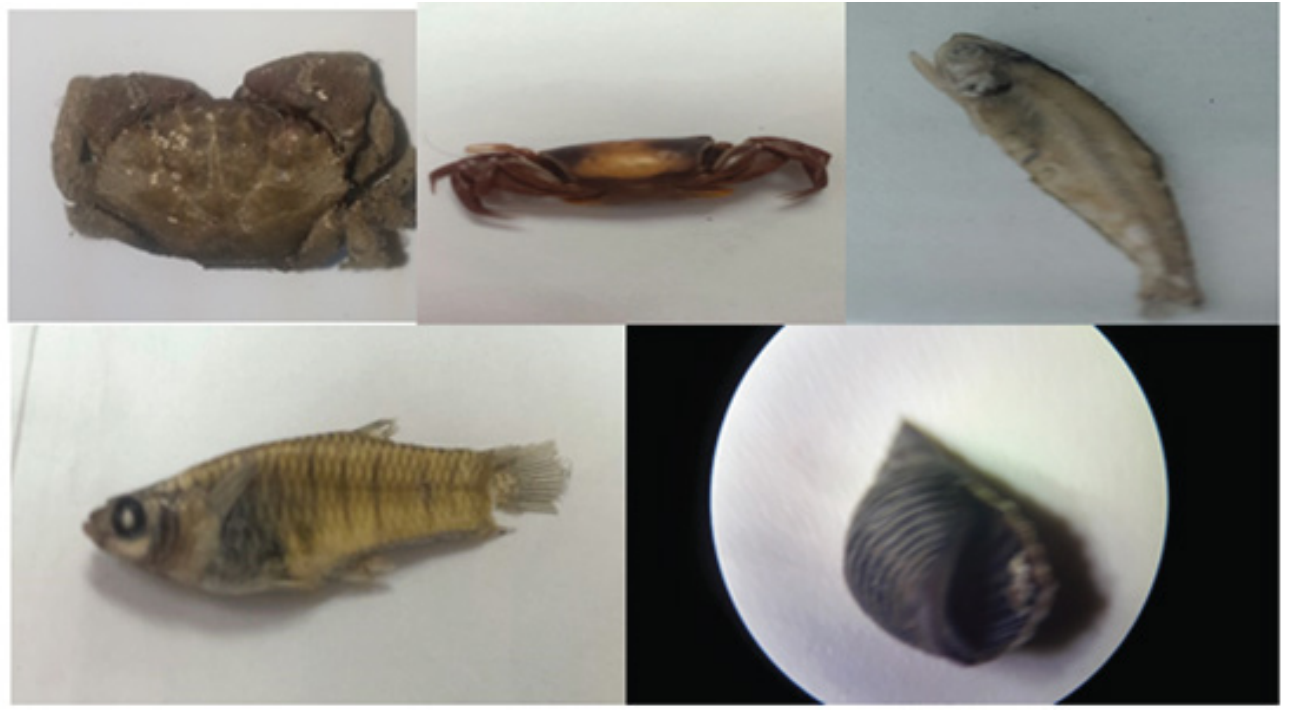

Fuente: Elaboración propia 


\section{CONCLUSIONES}

1. La diversidad y dominancia a través del índice de Simpson, muestran similitudes entre los dos esteros. Punto A obtuvo una dominancia baja $D^{\prime}=0.3469$ y una diversidad alta 1-D'= 0.6530 (Tabla 4), con respecto al Punto B no expreso diferencias significativas ya que este obtuvo dominancia baja $D^{\prime}=0.2$ y diversidad alta $1-D^{\prime}=$ 0.8 (Tabla 5), este último caracterizado por una mayor cantidad de familias presentes.

2. En el análisis de Shannon se observa que el valor más alto corresponde al Punto B con H'= 1.609437912 (Tabla 6), en tanto que Punto A obtuvo H'= 1.078992207 (Tabla 7). Punto A la distribución de los organismos no es igual ya que Anadara tuberculosa concentra tres organismos, por lo tanto hay una dominancia mayor y la equidad no es igual., aunque tengamos 7 individuos y 3 Familias este tiene un índice menor. Por ende Punto B tiene 5 individuos diferentes y 4 familias, índice de Shannon no cambia porque el número de especies es el mismo y la equidad es igual, ya que no está afectada por el número total de individuos colectados en el cuadrante.

3. Respecto a la Densidad Absoluta y Relativa se presenta mayor densidad por especie en Punto A, siendo Anadara Tuberculosa la especie más numerosa con una $D . A=0.6$ y $D . R=42.85 \%$ del total de especies, Iphigenia agior y Uca sp. Ambos con 2 organismos Con D.A= 0.4 y D.R= 28.57\% (Tabla 8). En Punto B presenta un total de 5 de organismos diferentes por lo tanto cada uno tiene una $D . A=0.2 \mathrm{Y}$ D. $R=20 \%$ (Tabla 9), Punto A fue el más dominante en cuanto a Densidad Absoluta (D.A), Punto B obtuvo una equidad exactamente igual tanto en D.A. y D.R.

4. En el estudio de comparación de comunidades biológicas de punto $A$ y punto $B$ se presenta una media de punto $A X=3.5$ y punto $B X=2.5$, siendo este el punto central del recorrido de una variable, una moda de punto $A M o=2$ y punto $B M o=$ 1 , que representa el dato que más se repite y una mediana de punto $A M=4$ y punto $B M=3$ que es valor de media cuando los datos son ordenados de acuerdo a su tamaño (Tabla 10). Estos datos se originan debido a los valores de las especies recolectadas en cada punto reflejando mayor media, moda y mediana en el Punto A debido a su mayor abundancia en especies de cada familia.

5. En cuanto a los parámetros biofísicos ( $\mathrm{pH}$ y Temperatura) no presento una diferencia significativa en cuanto a la densidad y variabilidad de especies, debido a que los 
resultados arrojados en ambos esteros, respecto a temperatura solo presento una diferencia de $1.3^{\circ} \mathrm{C}\left(34^{\circ} \mathrm{C}-35^{\circ} \mathrm{C}\right)$ y el pH de $0.2(7.02-7.06)$.

\section{DISCUSIONES}

1. En general el área estudiada correspondientes a dos comunidades biológicas marino costero, se observó que Punto $A$ fue donde se recolecto la mayor cantidad de individuos comparado al Punto $B$, ambos presentan una baja diversidad de especies, incluidas también las especies de importancia comercial, debido a los problemas de conservación que afectan dichas especies, donde el área no ha sido estudiada y la información es escasa, aunque la diversidad dependerá de la abundancia o dominancia de una especie en particular, el ambiente y la productividad de un ecosistema. (Berenice Licet, 2009, págs. 200-201). La baja densidad de organismos se puede dar por diferentes factores uno de los que más influye es la contaminación de los esteros, ya que obliga a los organismos a emigrar del estero contaminado a otros y aumenta la tasa de mortalidad.

2. La densidad de las especies con respecto al Índice de Shannon y Simpson no mostro diferencias significativas, sin embargo la diversidad total encontrada en Punto A en base al número de organismos colectados en esa zona costera fue alta en comparación con Punto $B$, esta diferencia puede deberse a las características del medio y la topografía de cada localidad, De tal forma que los patrones de densidad están influenciadas por tamaño, la disponibilidad de alimento y en la presión de depredación a la que se encuentra sujeta la especie. (Escalante, 2005, pág. 49), aunque las comunidades marinos costeras especialmente aquellas sometidas a actividades pesqueras como Litopenaeus Vannamei, están expuestos a impactos ambientales producidos por la acción del hombre , tanto en forma directa por medio de la pesca, como indirectamente del resultado de múltiples exposiciones a una variedad de contaminantes. (Marcelo A. Scelzo, 2002, pág. 58), por ello se debería de estudiar más a fondo las estructuras de las comunidades y los parámetros físico-químicos de la calidad de del agua con el objetivo de mitigar los impactos ambientales negativos en el entorno marino.

3. La diferencia generada entre la moda, media y mediana entra ambas áreas (Estero Tamasil y Estero Cabrera) en los cálculos se presenta una diferencia $=1$, no es una diferencia considerable pero que da conocer la abundancia de especies en cada punto de recolección al realizar la comparación de las comunidades biológi- 
cas presentes en cada área se presentó mayor abundancia el Punto A. Esta diferencia puede ser causada debido a que el punto A presenta las mejores condiciones físicas, químicas y biológicas y le brinda a las especies un mejor ambiente generándole las condiciones óptimas para el desarrollo y permitiéndoles su reproducción lo que aumenta la abundancia y riqueza de las especies esta área.

\section{BIBLIOGRAFÍA}

Aitana, P. 1. (s.f.). www.aitanatp.com. Recuperado el 03 de marzo de 2017, de www.aitanato.com: http://www.aitanatp.com/nivel6/ecosist/comunidad.htm

Berenice Licet, V. A. (2009). Contribución al conocimiento de los macromoluscos bentónicos asociados a la pepitona, Arca zebra (Swainson, 1833), del banco natural de Chacopata, Península de Araya, Venezuela. Scielo, 200-201.

Bojorges-Baños, J. C. (marzo de 2011). Riqueza y diversidad de especies de aves asociadas a manglar en tres sistemas lagunares en la region costera de Oaxaca, Mexico. scielo, 205-206.

Campos- bedolla, s. m. (2003). diccionario de biologia. D.F Mexico: Limusa.

earth, g. (9 de febrero de 2017). google earth. Obtenido de google earth: www.googleearth.com

ecured. (27 de marzo de 2015). https://www.ecured.cu/Zona_costera. Recuperado el 09 de abril de 2017, de https://www.ecured.cu/Zona_costera: www.ecured.cu/zona_costera

Escalante, T. H. (2005). DINÁMICA POBLACIONAL Y REPRODUCCIÓN DE LA ESTRELLA DE MAR PHATARIA UNIFASCIALIS (GRAY 1840) (ECHINODERMATA: ASTEROIDEA) EN PICHILINGUE, BAHÍA DE LA PAZ, BAJA CALIFORNIA SUR, MÉXICO. Scielo, 49.

FERNÁNDEZ, E. Á. (29 de enero de 2010). file:///C:/Users/MarC o \% $20 \mathrm{Nu} \%$ C $3 \%$ B 1 e z / D ownlo ad s / D i alnet - Molus cosCrustaceosYEquinodermosRestosDeOrigenMarin-3637707.pdf. DIALNET, 88-89. Recuperado el 29 de enero de 2017, de file://C:/Users/Marc o \% $20 \mathrm{Nu} \%$ C $3 \%$ B 1 e z / D ownlo ad s / D i alne t - Molus cosCrustaceosYEquinodermosRestosDeOrigenMarin-3637707.pdf: file:///C:/Users/Marco\% $20 \mathrm{Nu} \%$ C 3\% B 1 ez/Downloads/Dialnet-Molus cosCrustaceosYEquinodermosRestosDeOrigenMarin-3637707.pdf

Francisco Batres, J. A. (Enero de 2012). Ecologia Basica de los peces del Golfo de

Fonseca: bases para el manejo de la pesca artesanal. Academis EDU, 24, 26.

Graciela Ferrari, C. s. (2011). Estudio de las Comunidades Biológicas y Variables 
Abióticas en el tramo inferior del Rio Uruguay. MONTEVIDEO: UPM. Graciela Ferrari, C. s. (2012). Estudio de las Comunidades Biológicas y Variables Abióticas en el tramo inferiri del Rio Uruguay. MONTEVIDEO: UPM. Hart, R. D. (2014). Principios y conceptos de ecologia. Wikird, 9. Honduras Compras. (12 de febrero de 2012). Recuperado el www.hondurascompras.gob.hn de febrero de 2017

Jaime Ojeda, S. R. (diciembre de 2014). Patrones estacionales y espaciales de la diversidad de moluscos intermareales de bahia robalo, Canal Beagle, Reserva de la Biosfera Cabp de hornos, Chile. Revista de Biologia Marina y Oceanografia, 494-509.

José Joaquín Rodríguez, N. J. (1998). Análisis regional de la situación de la zona marina costera centroamericana.

Lizaralde, D. C. (2014). BIODIVERSIDAD Y ABUNDANCIA DE MACROINVERTEBRADOS BENTÓNICOS DE LA ZONA INTERMAREAL EN LA RESERVA DE PRODUCCIÓN FAUNISTTICA MARINO COSTERA PUNTILLADE SANTA ELENA LOS MESES DE NOVIEMBRE 2013 HASTA FEBRERO 2014. Universidad Estatal Peninsula de Santa Elena, Biologia Marina. Santa Elena: Santa Elena.

Lizarralde, D. C. (2015). BIODIVERSIDAD Y ABUNDANCIA DE MACROINVERTEBRADOS BENTÓNICOS DE LA ZONA INTERMAREAL EN LA RESERVA DE PRODUCCIÓN FAUNISTTICA MARINO COSTERA PUNTILLADE SANTA ELENA LOS MESES DE NOVIEMBRE 2013 HASTA FEBRERO 2014. Universidad de Santa Elena, Biologia Marina. Libertad, Ecuador: La Libertad: Universidad Estatal Península de Santa Elena, 2014.

Marcelo A. Scelzo, J. M. (2002). DINÁMICA POBLACIONAL Y REPRODUCCIÓN DE LA ESTRELLA DE MAR PHATARIA UNIFASCIALIS (GRAY 1840) (ECHINODERMATA: ASTEROIDEA) EN PICHILINGUE, BAHÍA DE LA PAZ, BAJA CALIFORNIA SUR, MÉXICO. Revista de Investigacion Desarrollo Pesquero, 58.

O. sanchez, M. H. (2007). Perspectivas sobre conservacion de ecosistemas acuaticos en Mexicos. D.F Mexico.

Perez, H. E. (2014). Biodiversidad de Peces en Mexico. Revista Mexicana de Biodiversidad, 1,2 .

Roca, J. M. (2015). VALORACIÓN DELA DIVERSIDAD DE MACROINVERTEBRADOSMARINOS BENTÓNICOS EN LA ZONA SUBMAREAL DE LA LIBERTAD SECTOR -LAESCOLLERAY LA CALETA DURANTE LOS MESES DE NOVIEMBRE 2014 -ABRIL 2015. Universidad Estatal Peninsula de Santa Elena, Biologia Marina. Santa Elena: Santa Elena.

Saldaña, A. (2013). Relación entre riqueza de especies y diversidad funcional de atributos foliares en dos ensambles de especies siempreverdes de un bosque templado lluvioso. Scielo, 178. Obtenido de file:///C:/Users/luis/Downloads/Infor- 
me-Final-de-Revision-del-Plan-de-Preparacion-y-Respuesta-Marcovia.pdf

Secretaría de Recursos Naturales y Ambiente de Honduras.Ministerio de Medio Ambiente y Recursos Naturales de El Salvador, M. d. (1998). Diagnostico del estado de los Recursos Naturales, socioeconomicos e institucionales de la zona costera del Golfo de Fonseca.

VM Zarco-Espinosa, J. V.-H.-P.-A. (2010). ESTRUCTURA Y DIVERSIDAD DE LA VEGETACIÓN ARBÓREA DEL PARQUE. Scielo, 6. 